\title{
Kernos
}

Revue internationale et pluridisciplinaire de religion grecque antique

$4 \mid 1991$

Varia

\section{À propos du sarcophage d'Aghia Triada : un rituel de nécromancie à l'époque protohistorique?}

\section{Robert Laffineur}

\section{OpenEdition \\ Journals}

\section{Édition électronique}

URL : http://journals.openedition.org/kernos/310

DOI : $10.4000 /$ kernos.310

ISSN : 2034-7871

\section{Éditeur}

Centre international d'étude de la religion grecque antique

\section{Édition imprimée}

Date de publication : 1 janvier 1991

Pagination : 277-285

ISSN : 0776-3824

\section{Référence électronique}

Robert Laffineur, «À propos du sarcophage d'Aghia Triada : un rituel de nécromancie à l'époque protohistorique? », Kernos [En ligne], 4 | 1991, mis en ligne le 11 mars 2011, consulté le 19 avril 2019. URL : http://journals.openedition.org/kernos/310 ; DOI : 10.4000/kernos.310 


\section{A PROPOS DU SARCOPHAGE D'AGHIA TRIADA : UN RITUEL DE NÉCROMANCIE À L'ÉPOQUE PRO'TOHISTORIQUE ?}

Le sarcophage en calcaire peint découvert en juillet 1903 par les fouilleurs italiens dans la chambre funéraire $n^{\circ} 4$ au nord-est de la villa minoenne d'Aghia Triada est sans doute un des documents de la protohistoire égéenne qui a suscité les plus fréquents commentaires et les plus nombreux essais d'interprétation ${ }^{1}$. La richesse iconographique de sa décoration, sa qualité, son bon état de préservation et la signification religieuse et funéraire des scènes représentées en faisaient, il est vrai, un témoin privilégié, comme aussi sa situation chronologique, à la fin de la période néopalatiale ou au début de la période postpalatiale, postérieurement à l'époque de floraison de la grande peinture minoenne. L'abondante bibliographie dont le monument a fait l'objet amènerait à douter de l'opportunité d'un nouvel examen ${ }^{2}$, d'autant qu'un document de cette importance, de surcroît unique en son genre, ne

1 Pour un historique des recherches consacrées au monument et une analyse détaillée de son décor figuré, on se reportera à la monographie exhaustive de Ch. R. Long, The Ayia Triadha Sarcophagus. A Study of Late Minoan and Mycenaean Funerary Practices and Beliefs, Göteborg, 1974 (SIMA, XLI), qui dispensera d'un inventaire des opinions émises depuis la découverte (l'ouvrage sera cité dans la suite LONG). Les interprétations plus récentes ont été mentionnées entre autres dans J. VANSCHOONWINKEL, La barque dans le culte et la religion créto-mycéniens, in Revue des archéologues et historiens d'art de Louvain, 15 (1982), p. 25-26. Pour une bonne illustration en couleurs, on renverra à Sp. MARINATOS et M. HIRMER, Kreta, Thera und das mykenische Hellas, Munich, 1973, pl. XXX-XXXПI, sans donner chaque fois dans la suite de l'exposé la référence précise à telle scène ou à tel détail.

2 L'interprétation peut cependant progresser, comme le montre la récente identification proposée pour les deux quadrupèdes attelés au char des «divinités» sur un des petits côtés. Voir T. Small, A Goat-Chariot on the Hagia Triada Sarcophagus, in AJA, 76 (1972), p. 327 et pl. 72, 1 et J.P. NAUERT, $A$ Goat-Chariot on the Hagia Triada Sarcophagus : a Further Note, in AJA, 76 (1972), p. 437. La question la plus complexe reste de savoir si les quatre panneaux expriment un thème unique et homogène. L'unité thématique n'est même pas assurée pour la scène de libation et la scène de présentation du long côté principal (LONG, p. 25). 
livre pas aisément, il faut l'avouer, la clé de son interprétation ${ }^{3}$. Je voudrais néanmoins, dans les quelques pages qui vont suivre, proposer une nouvelle lecture de la décoration figurée et tenter d'aboutir à une autre interprétation de l'ensemble du décor qui associerait les scènes des deux longs côtés.

J'attirerai d'abord l'attention sur un élément important de la décoration du sarcophage, à savoir la figure de l'extrémité droite du long panneau principal, qui montre une scène de libation et une "scène de présentation" ${ }^{4}$. Le caractère inhabituel de cette figure a été très tôt observé. L'absence de bras et la pose rigide contrastent singulièrement avec l'allure libre et naturelle des autres figures et donnent plutôt l'impression qu'il s'agit d'un personnage au corps emmailloté, voire d'une momie, et semblent bien obliger à exclure une identification comme divinité ${ }^{5}$. La taille réduite est également perceptible et paraît bien résulter d'un choix délibéré, puisque l'espace disponible au-dessus de la tête n'imposait pas une telle réduction ${ }^{6}$ et que toutes les autres figures du sarcophage occupent au contraire toute la hauteur du registre. La raison est alors sans doute, comme l'a suggéré Ch. Long, que l'artiste a voulu indiquer une figure dont le bas du corps est situé sous le niveau du sol ${ }^{7}$. On peut objecter que les équivalents d'une telle position ne sont pas fréquents ${ }^{8}$ ni bien identifiables et on peut être amené à douter davantage encore du bien-fondé de cette interprétation quand on se rappelle que la partie inférieure du corps a malheureusement disparu avec le coin inférieur droit de la scèn $e^{9}$. Mais la taille réduite me

3 Une même invitation à la prudence dans NAUERT, ibidem : «Because of the unique position of the Hagia Triada sarcophagus in Minoan art, any interpretation must exhibit restraint».

4 LONG, fig. 52, à droite. L'identification de ce long côté comme la face principale du sarcophage résulte de la position du monument dans la tombe : le panneau portant la scène de libation et la «scène de présentation" était dirigé vers le nord au moment de la fouille (donc au moins au cours de la dernière utilisation de la tombe) et il était donc le premier que l'on découvrait en pénétrant dans la chambre; cette paroi nord était en outre plus soigneusement dressée et l'enduit qui supportait la peinture y était disposé en une surface plus régulière (LONG, p. 25).

5 LONG, p. 45.

6 LONG, p. 44.

7 Ibidem.

8 Ils sont recensés dans LoNG, p. 44-45.

9 Voir le relevé original de la décoration reproduit dans M.P. NiLsson, The Minoan-Mycenaean Religion and its Survival in Greek Religion, Lund, 19502, fig. 196. 
semble un argument suffisant pour permettre de voir avec quelque certitude dans la figure de l'extrémité droite de la face principale la représentation du défunt dont le corps est partiellement enfoncé sous le sol. S'agit-il alors dans l'idée du peintre, comme le croit Ch. Long, de montrer l' "esprit» du mort en train de s'enfoncer dans le sol, en direction du monde souterrain, à l'image de l'esprit de Patrocle ${ }^{10}$ ? Cela implique la croyance en un au-delà souterrain, assurément vraisemblable, mais difficilement compatible, sur un même document en tout cas, avec la présentation d'un modèle réduit de barque, qui laisserait plutôt entrevoir la croyance en un au-delà transmarin ${ }^{11}$.

Une fois que l'on tient pour probable le principe de cet enfoncement partiel dans le sol, on pourrait toutefois aussi bien imaginer un mouvement en sens inverse et considérer que le défunt est représenté au contraire au moment où il émerge du sol. Une telle apparition se justifierait bien dans une illustration du rituel funéraire, même si elle témoigne de l'association sur une seule et même scène du monde de la réalité de l'exécution du rite et du monde surnaturel de l'effet qu'il est censé produire. Ne voit-on pas alors se présenter immédiatement à l'esprit l'épisode de l'évocation des morts, aux chants X et XI de l'Odyssée, où l'âme des défunts surgit des Enfers au travers d'une fosse creusée par le consultant? Et en poursuivant le parallèle entre certains détails de l'imagerie du sarcophage et les détails du rituel décrits dans le texte homérique, on ne peut manquer d'être frappé par les étroites similitudes entre les deux témoignages.

On a déjà évoqué plus haut l'enfoncement dans le sol, équivalent possible de la fosse que doit creuser Ulysse aussitôt qu'il est parvenu sur les rives de l'Achéron :

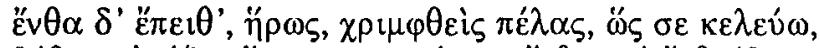

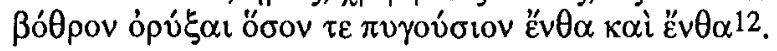

Attachons-nous à présent à la mention de la triple libation, de lait miellé, de vin doux et d'eau pure :

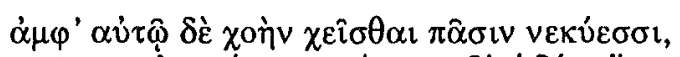

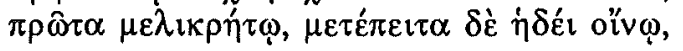

10 LONG, p. 46 (Iliade, XXIII, 100-101).

11 Voir en dernier lieu à ce propos R. LAFFineUR, La mer et l'au-delà dans l'Egée préhistorique, in Thalassa. L'Egée préhistorique et la mer. Actes de la $3 e$ Rencontre égéenne internationale de l'Université de Liège, Calvi, 23-25 auril 1990 (sous presse).

12 Odyssée, X, 516-517 et XI, 24-25. 


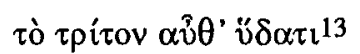

et observons que le long panneau principal montre précisément, dans sa partie gauche, deux femmes qui portent des seaux ou des baquets. La première verse le contenu du premier récipient dans un grand cratère, tandis que sa compagne attend de faire de même avec deux autres seaux suspendus aux extrémités d'une perche posée sur ses épaules. On s'est interrogé sur le contenu de ces baquets et on semble s'accorder pour y voir des récipients contenant un liquide. Le fin filet de couleur rose que l'on pouvait encore, au moment de la découverte, voir s'écouler du premier baquet, pourrait bien indiquer du vin ${ }^{14}$ et l'on est tenté d'imaginer que les deux autres contenaient le lait miellé et l'eau. Ces trois liquides seraient ensuite transportés, dans la partie centrale de la frise, par les trois porteurs, dans des récipients plus spécifiques, les deux premiers en forme de bovidé - des rhytons zoomorphes -, le troisième en forme de barque, avant que l'on ne procède aux libations proprement dites devant l'image du défunt. L'utilisation de rhytons est bien appropriée à une libation et l'on verrait volontiers dans les trois contenants les instruments d'une triple libation analogue à celle que doit exécuter le héros homérique. On observera cependant que la fonction première d'une barque n'est pas de contenir du liquide et que l'usage des modèles réduits d'embarcation comme rhytons n'est pas attesté en Égée, mais seulement à Chypre, au XIIe siècle ${ }^{15}$. On objectera surtout que si l'on voit ainsi dans les portions gauche et médiane de la frise deux épisodes successifs d'un même rituel, le mélange préalable des trois liquides dans le cratère ne permet pas de faire ensuite trois libations différentes, mais seulement trois fois la même libation et on comprend difficilement alors pourquoi on aurait figuré deux variétés de vase, deux rhytons zoomorphes et un modèle de barque. Si l'on considère en conséquence qu'il y a là au contraire deux rites distincts, une correspondance entre l'image et le texte n'est pas davantage évidente, à l'exception du nombre de porteurs et donc du nombre de libations qui vont s'accomplir. Faut-il associer la barque à l'eau ${ }^{16}$ et le lait à un des bovins ? Et quelle est alors

13 Odyssée, X, 518-520 et XI, 26-28.

14 LoNG, p. 36.

15 L. BASCH, Le musée imaginaire de la marine antique, Athènes, 1987, fig. 313316 (Lapithos).

16 Ou au miel mélangé au lait, par référence au curieux modèle de barque en terre cuite de la collection Mitsotakis dont le fond est pourvu d'un rayon de miel :

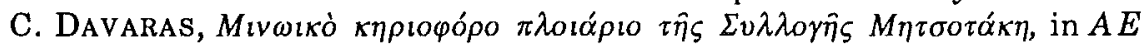
(1984), p. 55-95 et pl. 6. 
la raison de l'utilisation d'un second rhyton en forme de bovin pour une libation de vin ? Mais doit-on pousser aussi loin la comparaison et chercher une correspondance parfaite entre deux témoignages par ailleurs éloignés dans le temps?

Poursuivons plutôt la confrontation. Circé enjoint encore à Ulysse de sacrifier un agneau et une brebis

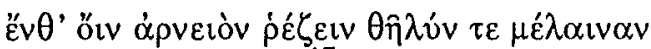

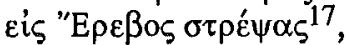

rite que l'on rapprochera du sacrifice de taureau illustré sur la face arrière du sarcophage d'Aghia Triada. La victime n'est certes pas la même - encore que deux petits capridés soient représentés sous la table à sacrifice et qu'Ulysse se voit conseiller, une fois rentré en Ithaque, de sacrifier en outre aux morts la meilleure de ses vaches ${ }^{18}$, mais un détail de la décoration du réceptacle funéraire mérite d'être observé en relation avec le rituel d'évocation des morts. Le sang de l'animal sacrifié s'écoule de manière très perceptible dans un récipient tronconique à une seule anse dans lequel on doit reconnaitre non pas un seau ou un baquet tronconique complet posé sur le sol mais plutôt la partie supérieure d'un rhyton-cornet à demi enfoncé dans le sol ${ }^{19}$. Cette figuration particulière a son correspondant exact sur un vase-anneau de Mycènes qui porte un accessoire à identifier sans le moindre doute comme la moitié supérieure d'un rhyton-cornet que l'on a voulu figurer enfoncé dans le sol ${ }^{20}$. Le sang de l'animal immolé peut ainsi pénétrer, par cet intermédiaire, dans la terre et on peut penser que son rôle est alors, comme dans le récit homérique, de se rapprocher des âmes des défunts qu'il est censé attirer vers la surface afin de permettre la consultation $^{21}$.

17 Odyssée, $\mathrm{X}, 527-528$.

18 Odyssée, X, 522-523 et XI, 30-31.

19 LONG, p. 63. Cet écoulement et cette dispersion dans la terre doit amener à écarter l'hypothèse originale de R. PARIBENI, reprise plus récemment par I. Sakellarakis (Das Kuppelgrab A von Archanes und das kretischmykenische Tieropferritual, in Prähistorische Zeitschrift, 45 [1970], p. 185 et 193), selon laquelle le sang du taureau serait recueilli dans le cratère de l'extrémité gauche de la face opposée.

20 LoNG, p. 63 et fig. 92. La surface de l'anneau, avec ses motifs de fleurettes peintes et son serpent plastique, représente vraisemblablement le sol.

21 J'avais interprété antérieurement cette pénétration du sang de la victime dans la terre comme un moyen de permettre au liquide de vie d'accomplir son action vivifiante dans la sépulture et comme une manifestation symbolique de 
Seule, finalement, l'offrande de farine que le héros doit exécuter dans la fosse ${ }^{22}$ n'est apparemment pas attestée dans l'iconographie du sarcophage, à moins qu'il ne faille interpréter de cette manière le geste du personnage féminin présent derrière la table de sacrifice ou reconnaître des pains ou des gâteaux dans les éléments contenus dans la sorte de panier visible au-dessus de l'autel en avant de la même scène ${ }^{23}$. Il reste aussi que la présence de musiciens sur les deux longs côtés du sarcophage - une joueuse de lyre derrière les deux femmes de la face principale et un joueur de double flûte près de la table à sacrifice - n'est pas mentionnée par le poète dans la description des rites préparatoires à la consultation des âmes des morts. Sur un plan plus général enfin, la localisation du rituel diffère assez sensiblement dans les deux cas. L'épisode odysséen se déroule en pleine nature, tandis que la représentation peinte a pour théâtre un environnement construit et aménagé composé d'un édicule à l'extrémité droite du registre, derrière l' "esprit" du défunt, d'un autel, d'une façade d'édifice cultuel avec cornes de consécration et de colonnes posées sur des bases et munies de couronnements en forme de double hache. L'ensemble évoquerait davantage, en moins monumental il est vrai, un véritable sanctuaire qui annoncerait le nécromanteion de l'Achéron de la période historique.

On sait que les vestiges mis au jour sur le site du nécromanteion d'Éphyra, au confluent de l'Achéron et du Cocyte, ne sont pas antérieurs

croyances en la régénération après la mort. L'alternative proposée aujourd'hui n'est peut-être pas entièrement incompatible avec une telle conception. Voir à ce propos R. LAFFineur, Fécondité et pratiques funéraires en Egée à l'âge du Bronze, in Archaeology and Fertility Cult in the Ancient Mediterranean. Papers presented at the First International Conference on Archaeology of the Ancient Mediterranean, Malta, 2-5 September 1985 (1986), p. $84-85$ et Weitere Beiträge zur Symbolik im mykenischen Bestattungsritual, in Kolloquium zur Ägäischen Vorgeschichte, Mannheim, 20.-22.2. 1986, Schriften des Deutschen Archäologen-Verbandes, IX (1987), p. 126-127.

22 Odyssée, X, 520 et XI, 28.

23 De même, le jet d'une pierre que le pèlerin devait sans doute accomplir afin de conjurer le mauvais sort - si du moins on doit interpréter comme le résultat de ces gestes répétés le tas de pierres découvert dans un couloir du nécromanteion d'Éphyra - n'a pas d'équivalent dans l'iconographie du sarcophage, pas plus que dans le texte homérique. À ce propos, S.I. DAKARIs, Das Taubenorakel von Dodona und das Totenorakel bei Ephyra, in Neue Ausgrabungen in Griechenland, Antike Kunst, 1. Beiheft (1963), 52. 
au IIIe siècle avant notre ère ${ }^{24}$, mais la haute antiquité de l'oracle y est attestée clairement par le témoignage d'Homère et celui d'Hérodote relatif à la consultation du tyran Périandre ${ }^{25}$, de même que par la trouvaille d'un lot de terres cuites des VIe et Ve siècles à l'image d'une divinité chthonienne ${ }^{26}$. Les environs immédiats ont livré également les traces d'une présence mycénienne non négligeable, en particulier l'enceinte cyclopéenne de Xylokastron, et le sanctuaire lui-même avait conservé quelques rares témoins d'une occupation au Bronze récent, qui devait sans doute être plus importante, mais que l'aménagement des bâtiments hellénistiques a fait presque entièrement disparaître ${ }^{27}$. S'il n'y a pas là l'indice d'une pratique cultuelle et oraculaire au IIe millénaire, la chose mérite d'être observée et elle a permis à $\mathrm{S}$. Dakaris de supposer que le culte chthonien avait selon toute vraisemblance été introduit en Thesprotie par des immigrants venus du nord-ouest du Péloponnèse, plus particulièrement d'Élide, où le culte d'Hadès est bien attesté, de même que les toponymes Éphyra et Achéron ${ }^{28}$.

On ne possède pas d'autres indices sur la possible existence de pratiques nécromantiques à l'époque mycénienne. La fouille conduite récemment par Th. Spyropoulos à Palaiokastro près de Gortys d'Arcadie a toutefois révélé une tombe à tholos munie d'un dispositif inhabituel : un opaion situé près du sommet et qui donne accès, vers l'intérieur, à une plate-forme délimitée par deux murets et supportée par une sorte d'exèdre. Le fouilleur est tenté de reconnaître là le premier nécromanteion mycénien parvenu jusqu'à nous ${ }^{29}$, mais il convient certes d'attendre un rapport quelque peu circonstancié de la découverte

24 Pour une synthèse des résultats des fouilles, voir DAKARIS, op. cit., p. 51-54 et Das Totenorakel am Acheron, in E. MÉlAs (ed.), Tempel und Stätten der Götter $25 \mathrm{~V}, 92$. Griechenlands, Cologne, 1977, p. 157-164.

26 S.I. DAKARIS, Das Taubenorakel von Dodona und das Totenorakel bei Ephyra, op. cit., p. 54 et pl. 24, 1-3; ID., Antiquité de l'Epire. Le nécromanteion de l'Achéron - Ephyra - Pandosia - Cassopè (Athènes, s.d.), p. 17 et fig. p. 18.

27 S.I. DAKaris, Das Totenorakel am Acheron, in E. MÉlas (ed.), Tempel und Stätten der Götter Griechenlands, Cologne, 1977, p. 164; ID., Antiquité de l'Epire, op. cit., p. 17-19.

28 Ibidem et ID., Das Taubenorakel von Dodona und das Totenorakel bei Ephyra, op. cit., p. 51. L'activité colonisatrice des Éléens dans la région se manifeste plus tard, vers 700, par la fondation de Pandosia. Il semble hasardeux de rattacher la division tripartite de la partie centrale du sanctuaire à une tradition protohistorique.

29 Voir la mention de la trouvaille dans N. PAPACHATZIS et I. LouCAS, Chronique des fouilles, in Kernos, 3 (1990), p. 367. 
avant de développer le rapprochement entre ce témoignage et la décoration du sarcophage d'Aghia Triada. Mais si l'on tient pour vraisemblable, provisoirement du moins, cette interprétation de Th. Spyropoulos et celle que je suggère ici, il faut noter dès à présent que dans les deux cas on entrevoit la perspective de l'existence d'un rituel lié au contexte funéraire qui n'est pas exécuté au moment de l'inhumation et qui n'est pas destiné au défunt mais qui est pratiqué de manière répétitive, régulièrement peut-être, durant la période qui suit la mise en terre et bénéficie avant tout aux vivants.

Que l'on ait là une manifestation de la persistance d'une tradition de l'Égée protohistorique dans le récit homérique n'a pas de quoi surprendre. Ce n'est qu'un exemple supplémentaire d'un phénomène bien attesté, malgré l'écart chronologique entre le Bronze récent et le haut archaïsme. Que cette tradition se prolonge au-delà, sur un peu plus d'un millénaire, jusqu'à l'époque hellénistique, dans les pratiques oraculaires du nécromanteion d'Éphyra est peut-être plus inattendu. Mais on sait qu'un culte a été pratiqué aux périodes historiques dans certaines tombes mycéniennes ${ }^{30}$ et des indices particulièrement évidents ont été observés dans ce sens récemment par G. St. Korres sur le site de la tombe à tholos de Voïdokilia en Messénie, pour l'époque hellénistique précisément. De nombreuses figurines et plaques de terre cuite mises au jour dans le secteur sud-est de la fouille et dans une petite chapelle hellénistique construite au nord-ouest témoignent, par leur iconographie, de l'existence d'un culte héroïque et chthonien à la fin du IVe siècle et au début du IIIe ${ }^{31}$. La relation de ce culte avec le monument funéraire mycénien n'est pas douteuse, pas plus que celle des restes d'un sacrifice de taureau, découverts par le premier fouilleur, Sp. Marinatos, dans les niveaux supérieurs à l'intérieur de la tombe et qui

30 Voir en particulier pour la Messénie l'étude exhaustive de G. St. KonRes, 'H

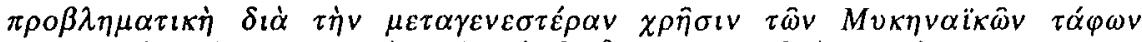

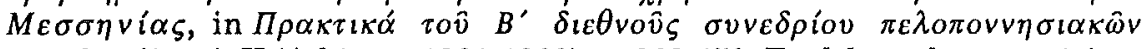

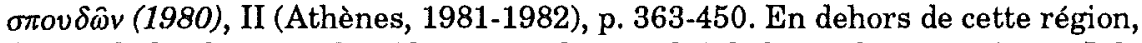
l'exemple le plus significatif est sans doute celui de la tombe mycénienne I de Thorikos, avec un dispositif archaique comprenant une table à offrandes et un bothros : J. SERVAIS, Le secteur mycénien sur le haut du Vélatouri, in Thorikos I 1963 (Gand, 1968), p. 30-31 et 37-39 (les trouvailles de poterie du même dépôt ont été étudiées depuis par M. DevilLers, An Archaic and Classical Votive Deposit from a Mycenaean Tomb at Thorikos, Miscellanea Graeca 8 [Gand, 1988]).

31 A propos de cette trouvaille et de son interprétation, G. St. Korres, Evidence for a Hellenistic Chthonian Cult in the Prehistoric Cemetery of Voidokoilia in Pylos (Messenia), in Klio, 70 (1988), p. 311-328. 
appartiennent à une période d'utilisation difficile à préciser mais en tout cas postérieure au IIe millénaire ${ }^{32}$.

L'évidence en faveur de la présente interprétation est assez limitée, on en conviendra. Elle ne me paraît pas cependant suffisamment négligeable pour renoncer à proposer une lecture des scènes principales du sarcophage d'Aghia Triada qui aille résolument dans une nouvelle direction ${ }^{33}$ et qui laisse entrevoir l'existence possible au Bronze récent de pratiques qui semblaient attestées seulement aux époques historiques. La démarche est sans doute hasardeuse, mais n'est-ce pas en définitive le sort de tout essai d'interprétation de documents figurés qui ne peuvent être éclairés par le témoignage de sources écrites contemporaines ou qui sont même simplement trop isolés pour pouvoir bénéficier du secours de l'examen comparatif ?

Université de Liège,

Robert LAFFINEUR

Place du XX-Août, 32,

B - 4000 LIÈGE

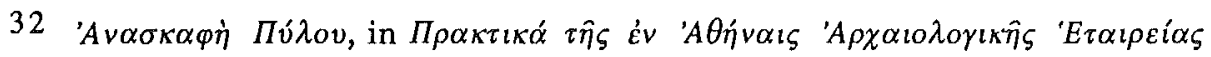
(1956), p. 203.

33 Les motifs des petits côtés du sarcophage ne s'inscrivent pas à l'évidence dans le schéma proposé. Les personnages en char, probablement des divinités, ne se laissent pas immédiatement identifier et les vestiges d'une procession qui subsitent à la partie supérieure du petit côté ouest (LONG, fig. 73) ne sont que très incomplètement conservés. 